\title{
Nanoscale
}

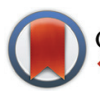

CrossMark

Cite this: Nanoscale, 2016, 8, 300

Received 17th September 2015, Accepted 13th November 2015 DOI: $10.1039 / \mathrm{c} 5 \mathrm{nr} 06419 \mathrm{~h}$ www.rsc.org/nanoscale

\section{Determining the 3D orientation of optically trapped upconverting nanorods by in situ single-particle polarized spectroscopy $\dagger$}

\author{
Paloma Rodríguez-Sevilla, ${ }^{a}$ Lucía Labrador-Páez, ${ }^{a}$ Dominika Wawrzyńczyk, ${ }^{b}$ \\ Marcin Nyk, ${ }^{\text {b Marek Samoć, }}$ ' Ajoy Kumar Kar, ${ }^{c}$ Mark D. Mackenzie, ${ }^{c}$ Lynn Paterson, ${ }^{d}$ \\ Daniel Jaque ${ }^{a}$ and Patricia Haro-González*a
}

\begin{abstract}
An approach to unequivocally determine the three-dimensional orientation of optically manipulated $\mathrm{NaYF}_{4}: \mathrm{Er}^{3+}, \mathrm{Yb}^{3+}$ upconverting nanorods (UCNRs) is demonstrated. Long-term immobilization of individual UCNRs inside single and multiple resonant optical traps allow for stable single UCNR spectroscopy studies. Based on the strong polarization dependent upconverted luminescence of UCNRs it is possible to unequivocally determine, in real time, their three-dimensional orientation when optically trapped. In single-beam traps, polarized single particle spectroscopy has concluded that UCNRs orientate parallel to the propagation axis of the trapping beam. On the other hand, when multiple-beam optical tweezers are used, single particle polarization spectroscopy demonstrated how full spatial control over UCNR orientation can be achieved by changing the trap-to-trap distance as well as the relative orientation between optical traps. All these results show the possibility of real time three-dimensional manipulation and tracking of anisotropic nanoparticles with wide potential application in modern nanobiophotonics.
\end{abstract}

\section{Introduction}

Rare earth doped Upconverting Nanoparticles (hereafter UCNPs) have emerged during the last few years as useful fundamental building blocks in modern photonics. UCNPs are being extensively used in a large variety of research fields including solar energy, nanomedicine and bioimaging, among many others. ${ }^{1-8}$ UCNPs show the unique capability of producing visible (VIS) luminescence under near-infrared (NIR) optical excitation by a multiphoton excitation process known as upconversion. ${ }^{6,9}$ When compared to other nanomaterials also capable of NIR-to-VIS optical conversion (such as metallic

\footnotetext{
${ }^{a}$ Fluorescence Imaging Group, Departamento de Física de Materiales, Modulo 4, Universidad Autónoma de Madrid C/Francisco Tomás y Valiente 7, Madrid, 28049, Spain.E-mail: patricia.haro@uam.es

${ }^{b}$ Advanced Materials Engineering and Modelling Group, Wroclaw University of Technology, Wyb. Wyspianskiego 27, 50-3 70 Wroclaw, Poland

${ }^{c}$ Institute of Photonics and Quantum Science, Heriot Watt University, Riccarton Campus, Edinburgh EH14 4AS, UK.E-mail: a.k.kar@hw.ac.uk ${ }^{d}$ Institute of Biological Chemistry, Biophysics and Bioengineering, School of Engineering and Physical Sciences, Heriot Watt University, Edinburgh, EH14 4AS, UK $\dagger$ Electronic supplementary information (ESI) available: Polarized emission of the colloidal suspension of $\mathrm{NaYF}_{4}: \mathrm{Er}^{3+}, \mathrm{Yb}^{3+}$ UCNRs, green emission band, optical force measurement and optical manipulation by two beam optical tweezers setup. See DOI: 10.1039/c5nr06419h
}

nanoparticles and semiconductor nanocrystals), UCNPs present several remarkable advantages including long-term photochemical stability, narrow emission lines, long luminescence lifetimes, and size-independent spectral shape of their luminescence bands. ${ }^{10-14}$ Recent studies have also pointed out that, since in UCNPs the multiphoton excitation is assisted by real electronic states, large NIR-to-VIS efficiencies could be achieved upon cw excitation without requiring the use of expensive, ultrafast, high power laser sources (as is the case when using gold nanoparticles or semiconductor nanocrystals). ${ }^{9}$ This fact boosted the use of UCNPs for optical conversion in solar cells as well as in the development of costeffective bioimaging equipment. ${ }^{15-17}$ In the particular field of fluorescence bioimaging, UCNPs are nowadays considered as one of the most promising in vitro luminescence probes because of the low autofluorescence generated by continuous wave NIR optical excitation. ${ }^{18}$ Moreover, recent studies have demonstrated that some UCNPs are not only capable of in vitro imaging but, in addition, they can also be used as intracellular thermal and chemical sensing units. ${ }^{19-22}$ In addition, intracellular studies based on single UCNP are nowadays a real possibility as the synthesis of ultra-small $(<10 \mathrm{~nm}$ in diameter) UCNPs showing large NIR-to-VIS optical conversion efficiencies has been recently demonstrated. ${ }^{23}$ As a consequence, UCNPs are also being considered as promising 
optical probes for experiments at single-cell and singleparticle levels.

As a result of the intensive research carried out on UCNPs during the last few years, a wide range of UCNPs consisting of different host lattices and dopants have appeared onto the scene. $^{4,24,25}$ Modifying the different synthesis parameters different emission bands, ${ }^{12}$ particle sizes ${ }^{23}$ and morphologies ${ }^{3,4,13,26-28}$ could be achieved. Among all of them, erbium and ytterbium codoped $\mathrm{NaYF}_{4}$ UCNPs are considered as the reference combination of dopants and host materials. ${ }^{13,23}$ In particular, the recently demonstrated possibility of synthesizing $\mathrm{NaYF}_{4}: \mathrm{Er}^{3+}, \mathrm{Yb}^{3+}$ upconverting nanorods (hereafter $\mathrm{NaYF}_{4}: \mathrm{Er}^{3+}, \mathrm{Yb}^{3+}$ UCNRs) appears to be especially interesting as they constitute a unique system that could be used in a wide range of applications including security inking, cell tracking, cell manipulation, and bottom-up fabrication of nanofluidic and optical devices. ${ }^{14,26,29,30}$

However, the full exploration of the potential applications of $\mathrm{NaYF}_{4}: \mathrm{Er}^{3+}, \mathrm{Yb}^{3+}$ UCNRs would require remote three-dimensional control of their spatial arrangement. Such control becomes mandatory for, as an example, the construction of $\mathrm{NaYF}_{4}: \mathrm{Er}^{3+}, \mathrm{Yb}^{3+}$ UCNR assemblies (interesting for security inking and nanofluidic applications) as well as for controlled incorporation of single $\mathrm{NaYF}_{4}: \mathrm{Er}^{3+}, \mathrm{Yb}^{3+}$ UCNRs into living cells. At present, several techniques have been demonstrated to be capable of remote manipulation and assembly of different nanorods. These include the application of electric and magnetic fields as well as the use of laminar flows in microfluidic channels. ${ }^{31-33}$ Despite the good results obtained so far by these techniques, they lack the ability to provide real time three-dimensional spatial control. Optical tweezers are an appealing tool for real time three-dimensional manipulation of nanosized objects in aqueous environments. ${ }^{34,35}$ First demonstrated by Ashkin and co-workers, Optical Trapping (OT) of sub-micrometric structures is based on the gradient force created in the surroundings of a tightly focused laser beam. ${ }^{36,37}$ When a nanoparticle is randomly moving close to the laser focus, the optical electric field polarizes the nanoparticle so that it behaves as an electric dipole in a non-homogeneous electric field. As a consequence, the polarized nanoparticle is pushed towards the beam focus. OT and remote manipulation of a great variety of different nanostructures (including metallic nanoparticles, semiconductor nanowires, semiconductor quantum dots (QDs) and, more recently, UCNPs) have been already demonstrated. ${ }^{37-43}$ In previous experiments, the characterization of the dynamics of trapped nanostructures has been limited to accurate measurements of both the trapping force and particle incorporation rates. Nevertheless, practical applications require a deep understanding of the exact orientation of the optically trapped nanoparticles inside the optical trap. This is a challenging task since conventional approaches cannot be used: the trapped particle size is well below the resolution provided by optical microscopy. Thus, the design and development of non-direct strategies for nanoparticle tracking inside optical traps becomes necessary. As an example, Jauffred et al. determined the position distribution curve of optically trapped QDs by accurate tracking of their two-photon excited emission. ${ }^{37}$ In the case of highly asymmetric nanoparticles (such as nanorods) not only the position inside the trap is relevant but also their intra-trap orientation needs to be known. Such knowledge has been achieved in the case of optically trapped Gold Nanorods (GNRs) and niobate nanowires by the real time analysis of luminescence and by polarized Second Harmonic Generation, respectively. These pioneering experiments have demonstrated that orientation of asymmetric nanoparticles inside optical traps is far from easy to predict. ${ }^{39,44}$ Niobate nanowires were found to align along the trapping beam axis whereas GNRs orientated parallel to the polarization vector of the trapping laser beam. It is also worth mentioning that, apart from the particle material, the characteristics of the trapped particle, such as its length in the case of nanorods, deeply affect the way it is trapped. ${ }^{45}$ Despite the interest from both fundamental and applied point of view neither optical trapping of UCNRs nor their intra-trap orientation has been demonstrated so far.

In this work, a single-beam OT setup has been used to demonstrate stable optical trapping of $\mathrm{NaYF}_{4}: \mathrm{Er}^{3+}, \mathrm{Yb}^{3+}$ UCNRs, with a highly anisotropic morphology. Resonant optical tweezers, in which the laser trapping radiation is partially absorbed by the optically trapped particle, allowed for real-time detection of the sequential incorporation of $\mathrm{NaYF}_{4}$ : $\mathrm{Er}^{3+}, \mathrm{Yb}^{3+}$ UCNRs into the optical trap by luminescence microscopy. Single $\mathrm{NaYF}_{4}: \mathrm{Er}^{3+}, \mathrm{Yb}^{3+} \mathrm{UCNR}$ spectroscopy in a dry environment was used to reveal the strong polarization dependence of the VIS luminescence generated by $\mathrm{NaYF}_{4}: \mathrm{Er}^{3+}$, $\mathrm{Yb}^{3+}$ UCNRs. This property has been further used, through the analysis of the polarization state of intra-trap luminescence, to unequivocally elucidate the equilibrium orientation of optically trapped $\mathrm{NaYF}_{4}: \mathrm{Er}^{3+}, \mathrm{Yb}^{3+}$ UCNRs with respect to the laser trapping beam.

\section{Experimental}

\section{Synthesis of $\mathrm{NaYF}_{4}: \mathrm{Er}^{3+}, \mathrm{Yb}^{3+}$ upconverting nanorods}

For the synthesis of $\mathrm{NaYF}_{4}: 2 \% \mathrm{Er}^{3+}, 18 \% \mathrm{Yb}^{3+}$ UCNRs we used a recently published protocol. ${ }^{46}$ The hydrothermal processing using microwave radiation (MAGNUM II ERTEC MV 02-02 reactor) was used to first prepare the lanthanide nitrate precursor salt $(1.2 \mathrm{mM})$, which was further mixed with $14 \mathrm{~mL}$ of deionized water, $0.7 \mathrm{~g},(17.5 \mathrm{mM})$ of $\mathrm{NaOH}, 7.1 \mathrm{~g},(22.6 \mathrm{mM})$ of oleic acid (90 wt\%), $10.0 \mathrm{~g}(21.7 \mathrm{mM})$ of ethanol and $0.3 \mathrm{~g}$ (7.2 $\mathrm{mM}$ ) of $\mathrm{NaF}$ to obtain a milky homogeneous solution. After 30 min of stirring at room temperature, the solution was transferred into a Teflon vessel, sealed and then placed in a microwave reactor, followed by $8.5 \mathrm{~h}$ of hydrothermal processing at $250{ }^{\circ} \mathrm{C}$, under average pressure lower than $25 \mathrm{~atm}$, the solution of $\mathrm{NaYF}_{4}: \mathrm{Er}^{3+}, \mathrm{Yb}^{3+}$ UCNRs was obtained. The synthesized $\mathrm{NaYF}_{4}: \mathrm{Er}^{3+}, \mathrm{Yb}^{3+}$ UCNRs were collected by centrifugation at $12000 \mathrm{rpm}$ for $15 \mathrm{~min}$, and washed twice with ethanol. The as-synthesized $\mathrm{NaYF}_{4}: 2 \% \mathrm{Er}^{3+}, 18 \% \mathrm{Yb}^{3+}$ UCNRs 
were dispersed in cyclohexane with a concentration of ca. $20 \mathrm{mg} \mathrm{mL}^{-1}$. In order to perform optical trapping experiments it was necessary to transfer $\mathrm{NaYF}_{4}: \mathrm{Er}^{3+}, \mathrm{Yb}^{3+}$ UCNRs to a distilled water solution, for which we used the ligand removal procedure reported by Bogdan et al. ${ }^{21}$ Briefly, $1 \mathrm{~mL}$ of $\mathrm{NaYF}_{4}$ : $\mathrm{Er}^{3+}, \mathrm{Yb}^{3+}$ UCNRs in cyclohexane was mixed with $10 \mathrm{ml}$ of deionized water for $2 \mathrm{~h}$, while the $\mathrm{pH}$ of the solution was set at 4 with $0.1 \mathrm{M}$ hydrochloric acid. After the reaction the aqueous solution was extracted with excess of cyclohexane to remove oleic acid molecules, further collected by centrifugation at $12000 \mathrm{rpm}$ for $15 \mathrm{~min}$, washed and finally dispersed in distilled water with concentration of $c a .7 \mathrm{mg} \mathrm{mL}{ }^{-1}$. Taking into account the mean size of a single $\mathrm{NaYF}_{4}: \mathrm{Er}^{3+}, \mathrm{Yb}^{3+}$ UCNR and the density of the bulk $\mathrm{NaYF}_{4}$ matrix the concentration of the solution was recalculated to be $\sim 10^{11} \mathrm{NRs}$ per $\mathrm{cm}^{3}$. Distilled water was used as a trapping solution since $\mathrm{NaYF}_{4}: \mathrm{Er}^{3+}, \mathrm{Yb}^{3+}$ UCNRs present a good colloidal character when dispersed in it without any evidence of agglomeration between the particles (see DLS measurements in Fig. S1†). Moreover, the low density of distilled water facilitates $\mathrm{NaYF}_{4}: \mathrm{Er}^{3+}, \mathrm{Yb}^{3+}$ UCNR manipulation when trapped since no strong optical forces are needed in order to overtake the drag force. At this point, we should note that distilled water shows a non-negligible absorption coefficient at the trapping wavelength $(980 \mathrm{~nm})$. This could lead to the appearance of thermal effects in the surroundings of the trap. According to previous studies, under our experimental conditions, we had estimated that the temperature increment induced by the focused laser radiation is lower than $5{ }^{\circ} \mathrm{C}$. We state that such reduced heating does not affect the optical trapping experiments. ${ }^{47}$

\section{Optical trapping experimental setup}

A single-beam optical tweezers setup is depicted in Fig. 1a. The $980 \mathrm{~nm}$ laser radiation coming from a single-mode fiber-

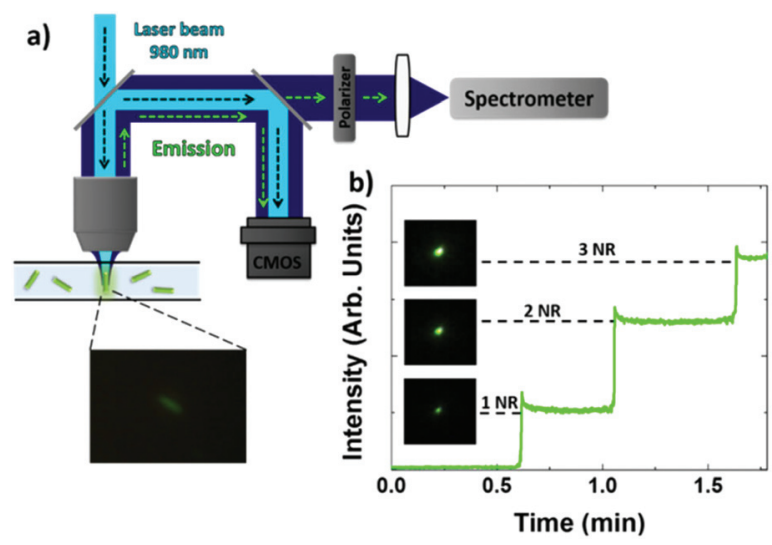

Fig. 1 (a). Schematic representation of the experimental setup. (b) Time evolution of the recovered luminescence at the trap position during multiple-particle trapping experiment. Sequential steps denote the incorporation of a single $\mathrm{NaYF}_{4}: \mathrm{Er}^{3+}, \mathrm{Yb}^{3+} \mathrm{UCNR}$ to the optical trap. Luminescence images show the change in the recovered intensity. coupled laser diode is collimated by a mounted Rochester aspheric lens (Thorlabs, A230TM-B) and then expanded by a $2 \times$ beam expander (Thorlabs, BE02M). This laser radiation is used for both optical trapping and to excite $\mathrm{NaYF}_{4}: \mathrm{Er}^{3+}, \mathrm{Yb}^{3+}$ UCNR luminescence. All the experiments were performed by using the maximum laser power provided by the laser $(60 \mathrm{~mW})$. For multiple-beam optical trapping experiments, the single-beam optical trapping setup was modified as schematically illustrated in Fig. S2. $\dagger$ We experimentally observed that the total laser power provided by the $980 \mathrm{~nm}$ laser diode was insufficient to form two highly stable optical traps by itself. A second $1064 \mathrm{~nm}$ laser beam (Nd:YAG laser, Ventus, Laser Quantum, Photonic Solutions) was used to create two additional traps perfectly overlapping with the $980 \mathrm{~nm}$ ones. The $1064 \mathrm{~nm}$ beam was made collinear with the $980 \mathrm{~nm}$ beam using a beamsplitter (Thorlabs, BS020) to combine the two beams (as seen in Fig. S2 $\dagger$ ). Then, the collinear laser beams were both split into two by using a second beamsplitter, and then reflected off plane mirrors to recombine them as shown in Fig. S2.† By slightly changing the alignment of either of the plane mirrors it was possible to create a slight angular difference in the propagating beams, resulting in two separate optical traps when focused through a high numerical aperture microscope objective. The laser power at any of these two traps $(80 \mathrm{~mW})$ is the sum of the overlapping 980 and $1064 \mathrm{~nm}$ laser powers. The extra contribution of the $1064 \mathrm{~nm}$ laser beam led to two stable optical traps. In summary, in this configuration, each optical trap is composed of the $1064 \mathrm{~nm}$ beam and the $980 \mathrm{~nm}$ laser beam; the more powerful laser operating at $1064 \mathrm{~nm}$ is used to provide the power for stable optical trapping, while the $980 \mathrm{~nm}$ radiation is needed for exciting $\mathrm{NaYF}_{4}$ : $\mathrm{Er}^{3+}, \mathrm{Yb}^{3+}$ UCNR luminescence (1064 $\mathrm{nm}$ absorption does not provide the sufficient emission from the trapped UCNR to perform accurate luminescence analysis). A $100 \times 1.25$ NA oilimmersed microscope objective was used for single $\mathrm{NaYF}_{4}$ : $\mathrm{Er}^{3+}, \mathrm{Yb}^{3+}$ UCNR experiments while a $10 \times 0.10 \mathrm{NA}$ microscope objective was used for spectral analysis of the $\mathrm{NaYF}_{4}: \mathrm{Er}^{3+}, \mathrm{Yb}^{3+}$ UCNRs suspension in order to ensure the analysis of multiple $\mathrm{NaYF}_{4}: \mathrm{Er}^{3+}, \mathrm{Yb}^{3+}$ UCNRs. For single $\mathrm{NaYF}_{4}: \mathrm{Er}^{3+}, \mathrm{Yb}^{3+}$ UCNR polarized emission experiments, a drop of the colloidal suspension of $\mathrm{NaYF}_{4}: \mathrm{Er}^{3+}, \mathrm{Yb}^{3+}$ UCNRs $\left(\sim 10^{11} \mathrm{NRs}\right.$ per $\left.\mathrm{cm}^{3}\right)$ was dried on a microscope slide and covered by a cover glass to prevent oil from coming into contact with the sample. For optical trapping experiments, a diluted colloidal suspension of $\mathrm{NaYF}_{4}: \mathrm{Er}^{3+}, \mathrm{Yb}^{3+}$ UCNRs $\left(\sim 5 \times 10^{9}\right.$ NRs per $\left.\mathrm{cm}^{3}\right)$ was placed inside a $100 \mu \mathrm{m}$ height microchannel (Ibidi Inc., $\mu$-Slide I 80106). For the analysis of the colloidal suspension of $\mathrm{NaYF}_{4}$ : $\mathrm{Er}^{3+}, \mathrm{Yb}^{3+}$ UCNRs $\left(\sim 10^{11} \mathrm{NRs}\right.$ per $\left.\mathrm{cm}^{3}\right)$, it was placed inside a spectrometer cuvette $(2 \mathrm{~mm} \times 10 \mathrm{~mm})$. For optical imaging a CMOS camera was used. Luminescence analysis was performed by optically coupling to the OT setup a high sensitivity Si CCD camera (Synapse, Horiba) attached to a monochromator (iH320, Horiba). At the entrance of the spectrometer a linear polarizer (Thorlabs, LPNIR050-MP2) mounted on a graduated wheel was placed for the selection of the polarized emission collected from the sample. 


\section{Results and discussion}

Fig. 2a shows a Transmission Electron Microscopy (TEM) image of the $\mathrm{NaYF}_{4}: \mathrm{Er}^{3+}, \mathrm{Yb}^{3+}$ UCNRs used all along this work. Statistical analysis of the TEM images provided the size histograms included in Fig. $2 \mathrm{~b}$ from which the $\mathrm{NaYF}_{4}: \mathrm{Er}^{3+}, \mathrm{Yb}^{3+}$ UCNR diameter and length were determined to be $140 \pm$ $20 \mathrm{~nm}$ and $1100 \pm 200 \mathrm{~nm}$, respectively. Details of the synthesis method and sample preparation can be found in the Experimental section. Additionally, the crystal structure and detailed spectroscopic characterization of the studied $\mathrm{NaYF}_{4}$ : $\mathrm{Er}^{3+}, \mathrm{Yb}^{3+}$ UCNRs are published elsewhere. ${ }^{46}$ Fig. $2 \mathrm{c}$ shows an optical image of an aqueous suspension of $\mathrm{NaYF}_{4}: \mathrm{Er}^{3+}, \mathrm{Yb}^{3+}$ UCNRs $(0.7 \mathrm{wt} \%)$ revealing the excellent colloidal character of our $\mathrm{NaYF}_{4}: \mathrm{Er}^{3+}, \mathrm{Yb}^{3+}$ UCNRs without any evident signs of precipitation during ensuing days. Fig. 2c also includes the optical image of the same cuvette containing the non-colored solution when optically excited by a $980 \mathrm{~nm}$ laser beam obtained by using a $600 \mathrm{~nm}$ long-pass filter. A clear red emission is observed that, according to the schematic energy level diagram shown in Fig. 2d, can be attributed to the ${ }^{4} \mathrm{~F}_{9 / 2} \rightarrow{ }^{4} \mathrm{I}_{15 / 2}$ transition of $\mathrm{Er}^{3+}$ ions, optically excited by an $\mathrm{Yb}^{3+} \rightarrow \mathrm{Er}^{3+}$ nonradiative energy transfer process.

\section{Dried sample experiments}

It is possible to find in the literature numerous examples revealing that highly anisotropic nanoparticles could generate a highly polarized luminescence. ${ }^{48-50}$ This was firstly demonstrated in non-spherical QDs that show well differentiated polarization states in their luminescence when their aspect ratios (length to diameter ratio) exceed $2 .{ }^{48}$ Later, a strong polarization anisotropy was discovered in codoped $\mathrm{Tm}^{3+}$ and a)

c)

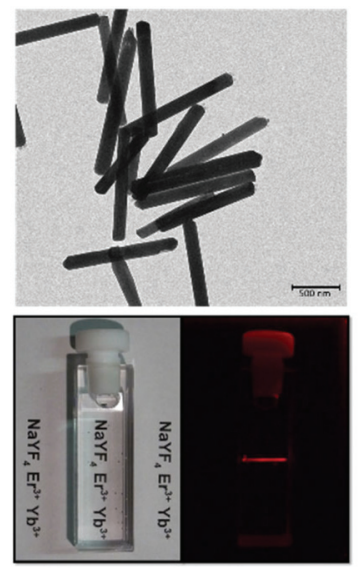

b)

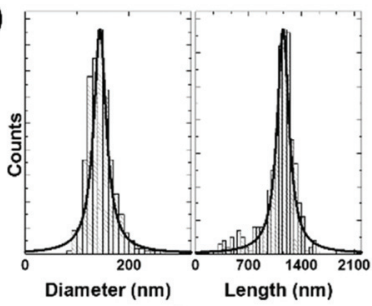

d)

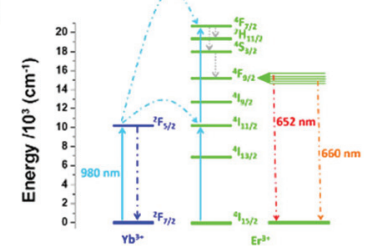

Fig. 2 (a) TEM image of the studied $\mathrm{NaYF}_{4}: \mathrm{Er}^{3+}, \mathrm{Yb}^{3+}$ UCNRs. (b) Size histograms obtained of TEM images representing diameter and length of the $\mathrm{NaYF}_{4}: \mathrm{Er}^{3+}, \mathrm{Yb}^{3+} \mathrm{UCNRs}$. (c) Optical images of the colloidal suspension of $\mathrm{NaYF}_{4}: \mathrm{Er}^{3+}, \mathrm{Yb}^{3+} \mathrm{UCNRs}$. The transparent colloidal suspension on the left presents a bright red emission when excited with a $980 \mathrm{~nm}$ laser beam. (d) Energy levels diagram of the upconversion energy transfer from $\mathrm{Yb}^{3+}$ to $\mathrm{Er}^{3+}$ ions. The transition of the emission levels of interest $(652$ and $660 \mathrm{~nm})$ in the ${ }^{4} \mathrm{~F}_{9 / 2}$ band are represented.
$\mathrm{Yb}^{3+} \mathrm{NaYF}_{4}$ nanorods with aspect ratios close to $7 .^{49}$ The exact origin of the polarization anisotropy in rare earth doped $\mathrm{NaYF}_{4}$ UCNRs is still unclear and, up to now, it is far from being fully understood. Indeed, prior to this work, it was not clear whether or not this phenomenon is restricted uniquely to the particular case of $\mathrm{Tm}^{3+}$ and $\mathrm{Yb}^{3+}$ ions. In order to check the possible polarization dependence of the upconversion luminescence generated by $\mathrm{NaYF}_{4}: \mathrm{Er}^{3+}, \mathrm{Yb}^{3+} \mathrm{UCNRs}$, we have performed single-particle polarized spectroscopy experiments. For this purpose, a drop of the colloidal suspension of $\mathrm{NaYF}_{4}: \mathrm{Er}^{3+}$, $\mathrm{Yb}^{3+}$ UCNRs was dried on a microscope slide and imaged by a CMOS camera. The luminescence of a single $\mathrm{NaYF}_{4}: \mathrm{Er}^{3+}, \mathrm{Yb}^{3+}$ UCNR immobilized on the surface of the microscope slide was achieved by focusing on it a continuous wave $980 \mathrm{~nm}$ laser beam by using a high numerical aperture (1.25 NA) microscope objective. The spot size of the $980 \mathrm{~nm}$ excitation beam was estimated to be $500 \mathrm{~nm}$ in radius. The visible luminescence generated by the $\mathrm{NaYF}_{4}: \mathrm{Er}^{3+}, \mathrm{Yb}^{3+}$ UCNR was collected by the same microscope objective and, after passing through different filters and confocal apertures, was spectrally analyzed with a CCD camera coupled to a high resolution spectrometer. In this work we have focused our attention on the ${ }^{4} \mathrm{~F}_{9 / 2} \rightarrow{ }^{4} \mathrm{I}_{15 / 2}$ red emission band as, under our experimental conditions, its intensity was several times higher than that of the green $\left({ }^{2} \mathrm{H}_{11 / 2},{ }^{4} \mathrm{~S}_{3 / 2} \rightarrow{ }^{4} \mathrm{I}_{15 / 2}\right)$ emission, as it can be observed in Fig. S3a of the ESI. $\dagger$ The polarization state of the NIR-excited VISluminescence, with respect to the $\mathrm{NaYF}_{4}: \mathrm{Er}^{3+}, \mathrm{Yb}^{3+}$ UCNR axis, was selected by placing a linear polarizer at the entrance of the spectrometer. Fig. 3a shows the normalized luminescence spectra generated by a single $\mathrm{NaYF}_{4}: \mathrm{Er}^{3+}, \mathrm{Yb}^{3+}$ UCNR corresponding to polarization states parallel and perpendicular to a)

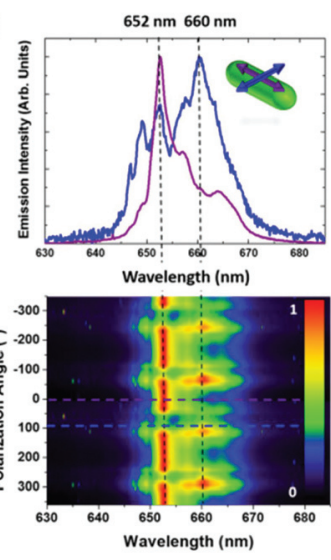

b)

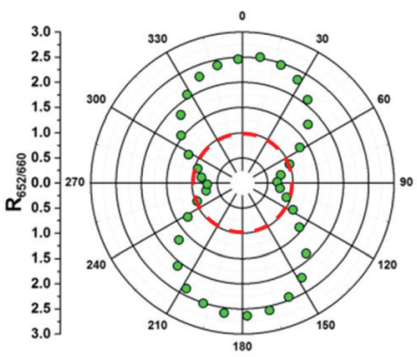

Fig. 3 (a) Emission spectra from a single $\mathrm{NaYF}_{4}: \mathrm{Er}^{3+}, \mathrm{Yb}^{3+}$ UCNR immobilized on a surface for two perpendicular emission polarization angles. Purple (blue) line represents the recovered emission parallel (perpendicular) to the optical axis of the $\mathrm{NaYF}_{4}: \mathrm{Er}^{3+}, \mathrm{Yb}^{3+} \mathrm{UCNR}$. Inset represents these two different polarizations of the electric field that gives the two distinct spectra. Two dimensional map represents the emission intensity of red band as a function of the emission polarization angle. (b) Polar plot of the ratio $\left(R_{652 / 660}\right)$ between 652 and 660 peak intensities as a function of the emission polarization angle. Red dashed circle delimits ratio values above and under 1 . 
the $\mathrm{NaYF}_{4}: \mathrm{Er}^{3+}, \mathrm{Yb}^{3+}$ UCNR axis (see inset in Fig. 3a). As can be observed, the luminescence intensities of the two main peaks (652 and $660 \mathrm{~nm}$ ) appear to be strongly polarization dependent. This is further evidenced in the two-dimensional map shown in Fig. 3a that includes the dependence of the emitted intensity as a function of the emission polarization angle (with respect to the $\mathrm{NaYF}_{4}: \mathrm{Er}^{3+}, \mathrm{Yb}^{3+} \mathrm{UCNR}$ axis). As can be seen, intensity peaks at $652 \mathrm{~nm}$ and $660 \mathrm{~nm}$ change with the polarization angle in a cyclic way. Fig. $3 \mathrm{~b}$ reveals that the intensity ratio between 652 and $660 \mathrm{~nm}$ peaks $\left(R_{652 / 660}\right)$ is hypersensitive to the polarization state. Values under and above 1 (inside and outside the red dashed circle) denote two polarization states, one dominated by the $652 \mathrm{~nm}$ peak intensity and a second one characterized by reaching the maximum intensity at $660 \mathrm{~nm}$. Note that the $R_{652 / 660}$ coefficient varies from 2.5 down to 0.5 when the polarization state is driven from being parallel to perpendicular to the $\mathrm{NaYF}_{4}: \mathrm{Er}^{3+}, \mathrm{Yb}^{3+} \mathrm{UCNR}$ axis. At this point we would like to mention that similar results were obtained when the ${ }^{2} \mathrm{H}_{11 / 2},{ }^{4} \mathrm{~S}_{3 / 2} \rightarrow{ }^{4} \mathrm{I}_{15 / 2}$ green emission was analyzed (see Fig. S4 in the ESI $\dagger$ ). The large induced polarization variation (five-fold) in the intensity ratio reveals the $R_{652 / 660}$ coefficient as a reliable and precise parameter to determine the relative orientation of $\mathrm{NaYF}_{4}: \mathrm{Er}^{3+}, \mathrm{Yb}^{3+}$ UCNRs by polarized singleparticle spectroscopy.

A tentative explanation of the polarized luminescence of $\mathrm{NaYF}_{4}: \mathrm{Er}^{3+}, \mathrm{Yb}^{3+}$ UCNRs can be given based on the recently published work of Ping Chen et al. ${ }^{51}$ In this work, the authors demonstrated that the polarized luminescence of Erbium doped $\mathrm{NaYF}_{4}$ nanodisks is due to the deterministic orientation of the crystallographic optical axis inside the nanoparticle. They claim that the optical axis in their nanostructures is perpendicular to the hexagonal (largest) face, so that the luminescence recorded along this direction does not show any polarization dependence. On the contrary, when measuring perpendicularly to this direction, due to the existence of an anisotropic crystal field, the luminescence becomes strongly polarization dependent due to the appearance of polarization induced forbidden and allowed transitions. ${ }^{52}$ We state at this point that, very likely, the optical axis of our $\mathrm{NaYF}_{4}: \mathrm{Er}^{3+}, \mathrm{Yb}^{3+}$ UCNRs lies parallel to their longest dimensions (i.e. optical axis coincides with the geometrical axis of the UCNRs). This would lead to a non-polarized luminescence when measured along the longest dimension (as it is observed later in the Optical trapping experiments section) and to a strongly polarized luminescence when spectra are recorded perpendicular to the longest dimension of the UCNR (see Fig. 3).

\section{Optical trapping experiments}

Single-beam resonant optical trapping. The experimental setup used for simultaneous luminescence tracking and OT of a single $\mathrm{Er}^{3+} / \mathrm{Yb}^{3+}$ codoped UCNR is schematically depicted in Fig. 1a. A detailed description of all the optical components can be found in the Experimental section. Briefly, a $980 \mathrm{~nm}$ laser beam was focused into a $100 \mu \mathrm{m}$ height microchannel, which contains a diluted colloidal suspension of $\mathrm{NaYF}_{4}: \mathrm{Er}^{3+}$, $\mathrm{Yb}^{3+}$ UCNRs, by using a 1.25 NA microscope objective. The
$980 \mathrm{~nm}$ laser beam, partially absorbed by $\mathrm{Yb}^{3+}$ ions inside the $\mathrm{NaYF}_{4}: \mathrm{Er}^{3+}, \mathrm{Yb}^{3+} \mathrm{UCNRs}$, was used to both trap the $\mathrm{NaYF}_{4}: \mathrm{Er}^{3+}$, $\mathrm{Yb}^{3+}$ UCNR and, simultaneously, to excite its luminescence when incorporated into the trap. The luminescence generated by the optically trapped $\mathrm{NaYF}_{4}: \mathrm{Er}^{3+}, \mathrm{Yb}^{3+} \mathrm{UCNR}$ was collected with the same objective used for focusing the $980 \mathrm{~nm}$ excitation laser beam, and it was spectrally analyzed by optically coupling the OT setup to the a high resolution spectrometer. Simultaneously, the luminescence image of the optical trap was recorded with a CMOS camera. A $700 \mathrm{~nm}$ short-pass filter was used to prevent the $980 \mathrm{~nm}$ radiation from reaching either the CMOS camera or the spectrometer. The sequential incorporation of individual $\mathrm{NaYF}_{4}: \mathrm{Er}^{3+}, \mathrm{Yb}^{3+} \mathrm{UCNRs}$ into the trap could be monitored in real time by recording the time evolution of the trap luminescence intensity by using the CMOS camera. A typical intensity profile is shown in Fig. 1b. From this intensity profile, it is possible to identify three sharp intensity steps at $0.6,1$ and $1.6 \mathrm{~min}$ after switching on the $980 \mathrm{~nm}$ laser beam. Each intensity step accounts for a single trapped $\mathrm{NaYF}_{4}: \mathrm{Er}^{3+}, \mathrm{Yb}^{3+} \mathrm{UCNR}$ whose luminescence is added to that generated by a previously trapped $\mathrm{NaYF}_{4}: \mathrm{Er}^{3+}, \mathrm{Yb}^{3+}$ UCNR. The luminescence images obtained after the incorporation of each single $\mathrm{NaYF}_{4}: \mathrm{Er}^{3+}, \mathrm{Yb}^{3+}$ UCNR are shown as the inset in Fig. 1b. At this point it should be noted that the time interval between consecutive incorporation of $\mathrm{NaYF}_{4}: \mathrm{Er}^{3+}, \mathrm{Yb}^{3+}$ UCNRs has been found to be strongly dependent on the concentration of $\mathrm{NaYF}_{4}: \mathrm{Er}^{3+}, \mathrm{Yb}^{3+}$ UCNRs in the suspension and by the $980 \mathrm{~nm}$ laser power, as it was already demonstrated to occur for spherical shaped $\mathrm{Er}^{3+} / \mathrm{Yb}^{3+}$ codoped UCNPs. ${ }^{53}$ The optical force acting on $\mathrm{NaYF}_{4}: \mathrm{Er}^{3+}, \mathrm{Yb}^{3+} \mathrm{UCNRs}$ was measured by applying the so-called hydrodynamic-drag method that is explained in detail in the ESI. $\dagger$ Fig. S5 $\uparrow$ includes the optical trapping force acting on a single $\mathrm{NaYF}_{4}: \mathrm{Er}^{3+}, \mathrm{Yb}^{3+} \mathrm{UCNR}$ as obtained at different $980 \mathrm{~nm}$ laser powers. The minimum laser power required for OT of a single $\mathrm{NaYF}_{4}: \mathrm{Er}^{3+}, \mathrm{Yb}^{3+} \mathrm{UCNR}$ under our experimental conditions was found to be $10 \mathrm{~mW}$. This value is 5 times lower than the corresponding one previously reported by us for spherical $\mathrm{Er}^{3+} / \mathrm{Yb}^{3+}$ codoped UCNPs. ${ }^{53}$ Fig. S5† clearly reveals that the optical force increases linearly with the $980 \mathrm{~nm}$ laser power as it was, indeed, expected. From the linear fit of experimental data we have obtained an optical trapping constant close to $k_{\text {trap }} \approx$ $2 \mathrm{pN} \mathrm{W}^{-1} \mu \mathrm{m}^{-1}$, which can be compared to that previously found for other UCNPs. ${ }^{54}$

Finally, it should be also mentioned that, although fluctuations in the position of $\mathrm{NaYF}_{4}: \mathrm{Er}^{3+}, \mathrm{Yb}^{3+}$ UCNRs could be taking place, the optically trapped $\mathrm{NaYF}_{4}: \mathrm{Er}^{3+}, \mathrm{Yb}^{3+}$ UCNRs remained stable inside the trap. This is evidenced by the intensity profile shown in Fig. $1 \mathrm{~b}$ in which sharp intensity drops down to the zero level (indicating that the $\mathrm{NaYF}_{4}: \mathrm{Er}^{3+}, \mathrm{Yb}^{3+}$ UCNRs have escaped from the trap and no luminescence is recorded) are not observed. This is in contrast to the results recently published by Jauffred et al. who evidenced the escape of QDs from an optical trap by the observation of intensity drops in the intensity time profiles. ${ }^{37}$ We state at this point that the larger size of our $\mathrm{NaYF}_{4}: \mathrm{Er}^{3+}, \mathrm{Yb}^{3+}$ UCNRs led to a 
reduced mobility as well as to a larger optical force in such a way that the optically trapped $\mathrm{NaYF}_{4}: \mathrm{Er}^{3+}, \mathrm{Yb}^{3+}$ UCNRs remain stable in the optical trap.

Once stable OT of UCNRs was experimentally achieved, the orientation of $\mathrm{NaYF}_{4}: \mathrm{Er}^{3+}, \mathrm{Yb}^{3+}$ UCNRs inside the trap was elucidated. This has been accomplished by performing polarized spectroscopy on a single optically trapped $\mathrm{NaYF}_{4}: \mathrm{Er}^{3+}, \mathrm{Yb}^{3+}$ UCNR. The emission spectra generated by an optically trapped $\mathrm{NaYF}_{4}: \mathrm{Er}^{3+}, \mathrm{Yb}^{3+}$ UCNR were systematically analyzed for different polarization states. At variance with the experimental results shown in Fig. 3, we observed that the luminescence spectra generated along the trapping direction was only slightly polarization dependent. This is evidenced in Fig. 4a that includes the emission spectra generated by a single optically trapped $\mathrm{NaYF}_{4}: \mathrm{Er}^{3+}, \mathrm{Yb}^{3+}$ UCNR as obtained for two arbitrary orthogonal polarizations. Within experimental error, they have been found to be virtually the same. In the emission spectra shown in Fig. 4a, the $660 \mathrm{~nm}$ line clearly dominates over the $652 \mathrm{~nm}$ peak, this being behavior independent of the polarization state as shown in the two-dimensional map where the emission intensity of the red band is represented as a function of the emission polarization angle. According to the data shown in Fig. 3, this fact suggests that the collected luminescence is polarized orthogonally to the axis of the trapped $\mathrm{NaYF}_{4}: \mathrm{Er}^{3+}, \mathrm{Yb}^{3+} \mathrm{UCNR}$. This unequivocally implies that the $\mathrm{NaYF}_{4}: \mathrm{Er}^{3+}, \mathrm{Yb}^{3+} \mathrm{UCNR}$ axis is parallel to the $k$ vector of the trapping laser beam, i.e. the $\mathrm{NaYF}_{4}: \mathrm{Er}^{3+}, \mathrm{Yb}^{3+} \mathrm{UCNR}$ is aligned parallel to the propagating direction of the trapping beam (see a)

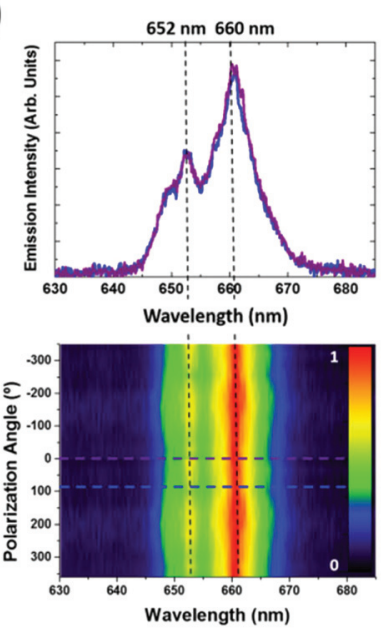

b)

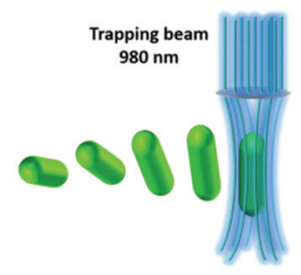

c)

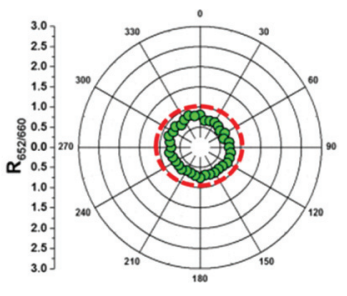

Fig. 4 (a) Emission spectra from a single optically trapped $\mathrm{NaYF}_{4}: \mathrm{Er}^{3+}, \mathrm{Yb}^{3+}$ UCNR for two arbitrary perpendicular emission polarization angles. Two dimensional map represents the emission intensity of the red band as a function of the emission polarization angle. (b) Schematic representation of the trajectory of a $\mathrm{NaYF}_{4}: \mathrm{Er}^{3+}, \mathrm{Yb}^{3+}$ UCNR moving towards the optical trap. $\mathrm{NaYF}_{4}: \mathrm{Er}^{3+}, \mathrm{Yb}^{3+} \mathrm{UCNR}$ longitudinal axis is parallel to the laser propagation direction when trapped. (c) Polar plot of the ratio between 652 and $660 \mathrm{~nm}$ peak intensities as a function of the emission polarization angle. Red dashed circle delimits ratio values above and below 1.
Fig. $4 \mathrm{~b})$. Fig. $4 \mathrm{c}$ shows the polar plot of the $R_{652 / 660}$ ratio with the emission polarization angle. When analyzing in detail the polar plot obtained for $R_{652 / 660}$, we observed that a nearly circular plot is obtained. This reveals that, when the luminescence is collected along the direction of the trapping beam, an isotropic scenario is obtained. This also concludes that the $\mathrm{NaYF}_{4}: \mathrm{Er}^{3+}, \mathrm{Yb}^{3+} \mathrm{UCNR}$ aligns parallel to the propagation direction of the trapping laser beam.

At this point it should be noted that near circular plot diagrams of the $R_{552 / 660}$ intensity ratio would be achieved if the $\mathrm{NaYF}_{4}: \mathrm{Er}^{3+}, \mathrm{Yb}^{3+}$ UCNR was orientated perpendicular to the trapping beam while rotating inside the trap with a rotation period much smaller than the acquisition time of luminescence spectra. In this scenario, the collected luminescence spectra result from a combination of the spectra obtained for the two polarization states shown in Fig. 3a, as it does occur when polarized spectroscopy is performed on a colloidal suspension of randomly oriented $\mathrm{NaYF}_{4}: \mathrm{Er}^{3+}, \mathrm{Yb}^{3+}$ UCNRs (see Fig. S3b in the ESI $\dagger$ ). Nevertheless, the emission spectra generated by an optically trapped $\mathrm{NaYF}_{4}: \mathrm{Er}^{3+}, \mathrm{Yb}^{3+}$ UCNR does not show any relevant contribution of parallel polarized emission. Thus, the rotation of $\mathrm{NaYF}_{4}: \mathrm{Er}^{3+}, \mathrm{Yb}^{3+}$ UCNR in a plane perpendicular to the laser trapping beam can be disregarded.

Multiple-beam optical trapping. The potential application of single particle polarized spectroscopy for three-dimensional monitoring of optically trapped UCNRs has been further explored by using a multiple-beam optical trap. A two beam optical tweezer setup was used to three dimensionally manipulate a single optically trapped $\mathrm{NaYF}_{4}: \mathrm{Er}^{3+}, \mathrm{Yb}^{3+} \mathrm{UCNR}$. For this purpose a modification of the optical tweezers setup depicted in Fig. 1a was used (for more details see the Experimental section and Fig. S2†). Such a setup allowed for an accurate control over the distance and relative position between the two optical traps. Two different situations were found to take place. When optical traps are separated by a distance longer than one micron (approximately), they behave as two independent single-beam optical traps. When a single $\mathrm{NaYF}_{4}: \mathrm{Er}^{3+}, \mathrm{Yb}^{3+}$ UCNR is optically trapped within one of the traps it aligns and remains parallel to the propagation axis of the beam (see Fig. 5a-c where a single UCNR is trapped in the bottom trap). Orientation of the UCNR is again evidenced by the luminescence spectrum in which the $652 \mathrm{~nm}$ and $660 \mathrm{~nm}$ emission peaks are of similar intensity, independent of the polarization angle. This is exactly the same result obtained in single-beam optical trap experiments. When the traps are close enough (distance between traps close to $1 \mu \mathrm{m}$ ), the trapped $\mathrm{NaYF}_{4}$ : $\mathrm{Er}^{3+}, \mathrm{Yb}^{3+} \mathrm{UCNR}$ is subjected to the force field resulting from the superposition of both traps. Under these conditions, the force field becomes anisotropic and, as a consequence, the $\mathrm{NaYF}_{4}: \mathrm{Er}^{3+}, \mathrm{Yb}^{3+}$ UCNR flips and orientates parallel to the line connecting both the traps. Thus, the proximity of both traps orientates the $\mathrm{NaYF}_{4}: \mathrm{Er}^{3+}, \mathrm{Yb}^{3+} \mathrm{UCNR}$ in the plane perpendicular to the trap beam propagation direction. This is evidenced in the fluorescence image shown in Fig. $5 \mathrm{~d}$ as well as in the associated single-particle luminescence spectrum acquired 


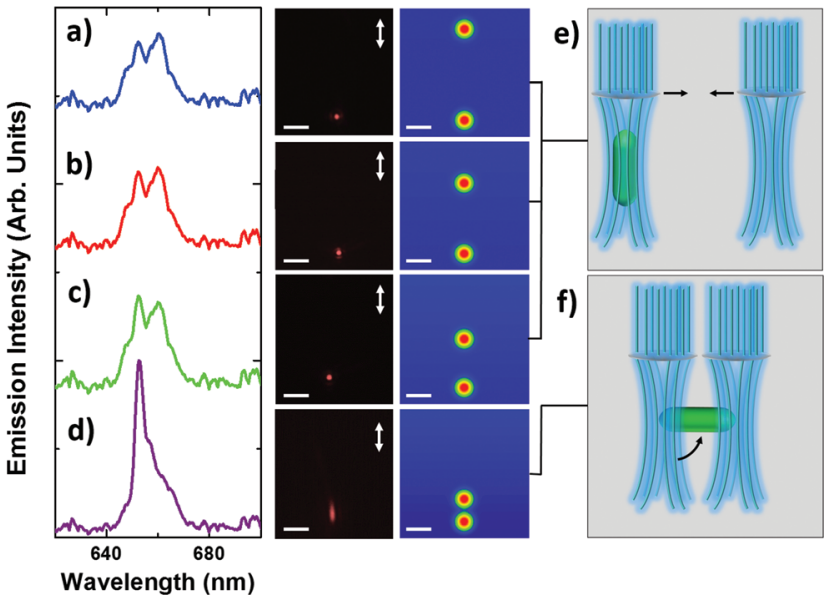

Fig. 5 Evolution of the emitted spectra of a trapped $\mathrm{NaYF}_{4}: \mathrm{Er}^{3+}, \mathrm{Yb}^{3+}$ UCNR when the distance between traps is reduced. The recorded emission polarization angle is fixed to that indicated by white arrows. It can be seen that the emission spectra changes from that attributed to perpendicular polarization $(a-c)$ to that for the parallel polarization state (d). Scale bar corresponds to $1 \mu \mathrm{m}$ in all images. Panels $(e-f)$ are schematic representations of the two $\mathrm{NaYF}_{4}: \mathrm{Er}^{3+}, \mathrm{Yb}^{3+} \mathrm{UCNR}$ positions.

with the selection polarized parallel to the inter-trap connection line (see white arrows in Fig. 5a-d that indicate the analyzed polarization angle). Under these conditions, the emission spectrum is dominated by the $652 \mathrm{~nm}$ emission line indicating that the $\mathrm{NaYF}_{4}: \mathrm{Er}^{3+}, \mathrm{Yb}^{3+} \mathrm{UCNR}$ is aligned parallel to the polarizer direction, i.e. the $\mathrm{NaYF}_{4}: \mathrm{Er}^{3+}, \mathrm{Yb}^{3+} \mathrm{UCNR}$ is aligned parallel to the inter-trap connection line. The relative orientation of the UCNR and the two traps is schematically shown in Fig. 5e and f.

Further manipulation of the two trap positions by keeping this inter-trap distance close to $1 \mu \mathrm{m}$ allows, in principle, for full control/rotation of the $\mathrm{NaYF}_{4}: \mathrm{Er}^{3+}, \mathrm{Yb}^{3+} \mathrm{UCNR}$ within the plane perpendicular to the laser beams' direction. This possibility has been demonstrated by rotating one trap around the other by $90^{\circ}$ and keeping the inter-trap distance fixed at $1 \mu \mathrm{m}$ (see schematic drawings in the right column of Fig. 6). Rotation of the optically trapped UCNR is shown by the fluorescence images in the central column of Fig. 6 . This in-plane rotation is further evidenced by the changes observed in the spectral shape of the polarized upconversion spectrum obtained by keeping the measured polarization angle fixed as indicated by arrows shown in Fig. 6. Rotation of the optical traps causes a shift from an emission spectrum dominated by the $652 \mathrm{~nm}$ emission line to an emission spectrum in which both 652 and $660 \mathrm{~nm}$ emission lines are of comparable intensity. This unequivocally reveals a complete $90^{\circ}$ in-plane rotation of the $\mathrm{NaYF}_{4}: \mathrm{Er}^{3+}, \mathrm{Yb}^{3+}$ UCNR. Note that the data shown in Fig. 5 and 6 do not only reveal the possibility of accurate three-dimensional optical manipulation of single UCNRs but also the continuous monitoring of their three-dimensional orientation by single particle spectroscopy.

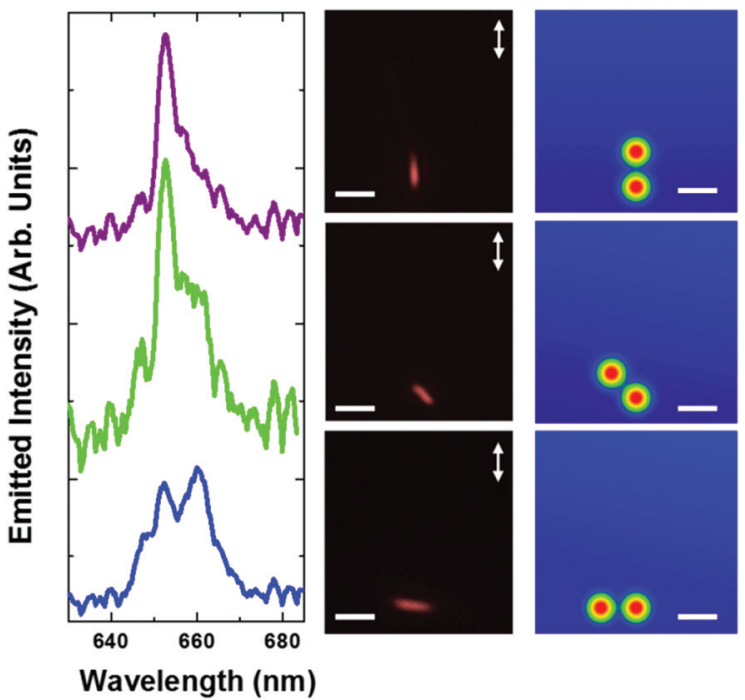

Fig. 6 Evolution of the emitted spectra when rotating the longitudinal axis of the $\mathrm{NaYF}_{4}: \mathrm{Er}^{3+}, \mathrm{Yb}^{3+} \mathrm{UCNR}$. Emission polarization angle is fixed to that indicated by white arrows. Perpendicular polarization is measured when the $\mathrm{NaYF}_{4}: \mathrm{Er}^{3+}, \mathrm{Yb}^{3+} \mathrm{UCNR}$ axis happen to be perpendicular to the emission polarization angle (lower panel). An intermediate state is observed when the $\mathrm{NaYF}_{4}: \mathrm{Er}^{3+}, \mathrm{Yb}^{3+} \mathrm{UCNR}$ axis form approximately $45^{\circ}$ with the emission polarization angle and both polarization states are measured (middle panel). Scale bar corresponds to $1 \mu \mathrm{m}$ in all images.

\section{Conclusions}

In summary, resonant stable optical trapping of $\mathrm{NaYF}_{4}: \mathrm{Er}^{3+}$, $\mathrm{Yb}^{3+}$ upconverting nanorods has been demonstrated. The efficient excitation of the $\mathrm{Er}^{3+}$ visible luminescence by the partially absorbed $980 \mathrm{~nm}$ trapping radiation of the $\mathrm{Yb}^{3+}$ ions, allowed for real time tracking of the optically trapped $\mathrm{NaYF}_{4}$ : $\mathrm{Er}^{3+}, \mathrm{Yb}^{3+}$ upconverting nanorods by simple luminescence imaging microscopy. The time evolution of the luminescence images from the optical trap evidenced the sequential incorporation of single $\mathrm{NaYF}_{4}: \mathrm{Er}^{3+}, \mathrm{Yb}^{3+}$ upconverting nanorods as well as their stable localization inside the trap. Single-particle polarized spectroscopy experiments performed on immobilized $\mathrm{NaYF}_{4}: \mathrm{Er}^{3+}, \mathrm{Yb}^{3+}$ upconverting nanorods revealed that their two-photon excited luminescence is strongly polarized, evidence for the existence of two well-defined polarization states corresponding to the polarization of luminescence perpendicular and parallel to the axis of $\mathrm{NaYF}_{4}: \mathrm{Er}^{3+}, \mathrm{Yb}^{3+}$ upconverting nanorods. This strong polarization dependence of luminescence has been used to unequivocally determine the orientation of optically trapped $\mathrm{NaYF}_{4}: \mathrm{Er}^{3+}, \mathrm{Yb}^{3+}$ upconverting nanorods inside single and double beam optical traps. It has been concluded that, under our experimental conditions, $\mathrm{NaYF}_{4}: \mathrm{Er}^{3+}, \mathrm{Yb}^{3+}$ upconverting nanorods orientated parallel to the propagation direction of the trapping laser beam in a single-beam optical tweezers setup. Two beam optical trapping allowed for controlled three-dimensional manipulation of single $\mathrm{NaYF}_{4}: \mathrm{Er}^{3+}, \mathrm{Yb}^{3+}$ upconverting nanorod whose threedimensional orientation was unequivocally determined by the 
analysis of the polarization state of their single particle luminescence.

The possibility, demonstrated here, of applying simple experimental approaches to achieve three-dimensional manipulation of the upconverting nanorods, whilst knowing their exact position and orientation, opens up a new avenue into their use in numerous applications ranging from biology to nanofluidics.

\section{Authors contribution}

The manuscript was written through contributions of all the authors. All the authors have given approval to the final version of the manuscript.

\section{Conflict of interest}

The authors declare no competing financial interest.

\section{Acknowledgements}

This work was supported by the Spanish Ministerio de Educación y Ciencia (MAT2013-47395-C4-1-R) and by Banco Santander for "proyectos de cooperación interuniversitaria" (2015/ ASIA/06). Patricia Haro-González thanks the Spanish Ministerio de Economía y Competitividad (MINECO) for the Juan de la Cierva program. Paloma Rodríguez-Sevilla thanks the Spanish Ministerio de Economía y Competitividad (MINECO) for the "Promoción del talento y su Empleabilidad en $\mathrm{I}+\mathrm{D}+\mathrm{i}$ " statal program. Dominika Wawrzyńczyk, Marcin Nyk and Marek Samoć acknowledge the support from the National Science Centre under grant DEC-2012/04/M/ST5/00340, by a statutory activity subsidy from the Polish Ministry of Science and Higher Education for the Faculty of Chemistry of Wroclaw University of Technology, as well as from the Foundation for Polish Science under START 2014 programme. This work has received funding from The Royal Society (IE130466). Mark D. Mackenzie acknowledges funding from EPSRC (grant no $\mathrm{EP} / \mathrm{J} 500227 / 1)$.

\section{Notes and references}

1 A. P. Bartko, L. A. Peyser, R. M. Dickson, A. Mehta, T. Thundat, R. Bhargava and M. D. Barnes, Chem. Phys. Lett., 2002, 358, 459-465.

2 M. Gregor, A. Kuhlicke and O. Benson, Opt. Express, 2009, 17, 24234-24243.

3 W. Gao, S. Sattayasamitsathit, J. Orozco and J. Wang, J. Am. Chem. Soc., 2011, 133, 11862-11864.

4 S. Hao, G. Chen and C. Yang, Theranostics, 2013, 3, 331345.

5 Y. Zhang, W. Wei, G. K. Das and T. T. Y. Tan, J. Photochem. Photobiol., C, 2014, 20, 71-96.
6 W. Zheng, P. Huang, D. Tu, E. Ma, H. Zhu and X. Chen, Chem. Soc. Rev., 2015, 44, 1379-1415.

7 J. C. Goldschmidt and S. Fischer, Adv. Opt. Mater., 2015, 3, 510-535.

8 C. Miao, C. Chen, Q. L. Dai, L. Xu and H. W. Song, J. Colloid Interface Sci., 2015, 440, 162-167.

9 L. M. Maestro, E. M. Rodriguez, F. Vetrone, R. Naccache, H. L. Ramirez, D. Jaque, J. A. Capobianco and J. G. Solé, Opt. Express, 2010, 18, 23544-23553.

10 L. Martinez Maestro, E. Martin Rodriguez, F. Vetrone, R. Naccache, H. Loro Ramirez, D. Jaque, J. A. Capobianco and J. Garcia Sole, Opt. Express, 2010, 18, 23544-23553.

11 F. Wang, Y. Han, C. S. Lim, Y. Lu, J. Wang, J. Xu, H. Chen, C. Zhang, M. Hong and X. Liu, Nature, 2010, 463, 10611065.

12 A. Bednarkiewicz, D. Wawrzynczyk, M. Nyk and M. Samoc, J. Rare Earths, 2011, 29, 1152-1156.

13 M. Ding, S. Yin, D. Chen, J. Zhong, Y. Ni, C. Lu, Z. Xu and Z. Ji, Appl. Surf. Sci., 2015, 333, 23-33.

14 F. M. Mor, A. Sienkiewicz, L. Forró and S. Jeney, ACS Photonics, 2014, 1, 1251-1257.

15 F. Wang, D. Banerjee, Y. S. Liu, X. Y. Chen and X. G. Liu, Analyst, 2010, 135, 1839-1854.

16 R. Naccache, F. Vetrone and J. A. Capobianco, ChemSusChem, 2013, 6, 1308-1311.

17 X. Xie and X. Liu, Nat. Mater., 2012, 11, 842-843.

18 K. König, J. Microsc., 2000, 200, 83-104.

19 D. Jaque, B. del Rosal, E. Martin Rodriguez, L. Martinez Maestro, P. Haro-Gonzalez and J. Garcia Sole, Nanomedicine, 2014, 9, 1047-1062.

20 D. Jaque and F. Vetrone, Nanoscale, 2012, 4, 4301-4326.

21 N. Bogdan, F. Vetrone, G. A. Ozin and J. A. Capobianco, Nano Lett., 2011, 11, 835-840.

22 G. Chen, H. Qiu, P. N. Prasad and X. Chen, Chem. Rev., 2014, 114, 5161-5214.

23 D. J. Gargas, E. M. Chan, A. D. Ostrowski, S. Aloni, M. V. P. Altoe, E. S. Barnard, B. Sanii, J. J. Urban, D. J. Milliron, B. E. Cohen and P. J. Schuck, Nat. Nanotechnol., 2014, 9, 300-305.

24 A. Gnach and A. Bednarkiewicz, Nano Today, 2012, 7, 532563.

25 G. S. Yi, H. C. Lu, S. Y. Zhao, G. Yue, W. J. Yang, D. P. Chen and L. H. Guo, Nano Lett., 2004, 4, 2191-2196.

26 Y. Zhang, L. Zhang, R. Deng, J. Tian, Y. Zong, D. Jin and X. Liu, J. Am. Chem. Soc., 2014, 136, 4893-4896.

27 L. Zhihua, L. Ying, W. Yanan, M. Haixia, D. Yu and L. Hong, J. Alloys Compd., 2014, 613, 18-24.

28 Z.-Q. Liang, S.-L. Zhao, Y. Cui, L.-J. Tian, J.-J. Zhang and Z. Xu, Chin. Phys. B, 2015, 24, 037801.

29 R. Karnik, R. Fan, M. Yue, D. Li, P. Yang and A. Majumdar, Nano Lett., 2005, 5, 943-948.

30 D. J. Sirbuly, M. Law, H. Yan and P. Yang, J. Phys. Chem. B, 2005, 109, 15190-15213.

31 P. A. Smith, C. D. Nordquist, T. N. Jackson, T. S. Mayer, B. R. Martin, J. Mbindyo and T. E. Mallouk, Appl. Phys. Lett., 2000, 77, 1399-1401. 
32 M. Chen, L. Sun, J. E. Bonevich, D. H. Reich, C. L. Chien and P. C. Searson, Appl. Phys. Lett., 2003, 82, 3310-3312.

33 B. Messer, J. H. Song and P. Yang, J. Am. Chem. Soc., 2000, 122, 10232-10233.

34 K. Svoboda and S. M. Block, Annu. Rev. Biophys. Biomol. Struct., 1994, 23, 247-285.

35 J. E. Molloy and M. J. Padgett, Contemp. Phys., 2002, 43, 241-258.

36 A. Ashkin, Biophys. J., 1992, 61, 569-582.

37 P. M. Bendix, L. Jauffred, K. Norregaard and L. B. Oddershede, IEEE J. Sel. Top. Quantum Electron., 2014, 20, 15-26.

38 M. Dienerowitz, M. Mazilu and K. Dholakia, NANOP, 2008, 2, 021875-021832.

39 M. Pelton, M. Liu, H. Y. Kim, G. Smith, P. Guyot-Sionnest and N. F. Scherer, Opt. Lett., 2006, 31, 2075-2077.

40 C. Selhuber-Unkel, I. Zins, O. Schubert, C. Sonnichsen and L. B. Oddershede, Nano Lett., 2008, 8, 2998-3003.

41 P. J. Reece, W. J. Toe, F. Wang, S. Paiman, Q. Gao, H. H. Tan and C. Jagadish, Nano Lett., 2011, 11, 2375-2381.

42 F. Wang, W. J. Toe, W. M. Lee, D. McGloin, Q. Gao, H. H. Tan, C. Jagadish and P. J. Reece, Nano Lett., 2013, 13, 1185-1191.

43 P. Rodríguez-Sevilla, H. Rodríguez-Rodríguez, M. Pedroni, A. Speghini, M. Bettinelli, J. G. Solé, D. Jaque and P. HaroGonzález, Nano Lett., 2015, 15, 5068-5074.

44 R. Grange, F. Dutto and A. Radenovic, Niobates nanowires: synthesis, characterization and applications, In Tech, 2011.
45 A. Irrera, P. Artoni, R. Saija, P. G. Gucciardi, M. A. Iatì, F. Borghese, P. Denti, F. Iacona, F. Priolo and O. M. Maragò, Nano Lett., 2011, 11, 4879-4884.

46 D. Wawrzynczyk, D. Piatkowski, S. Mackowski, M. Samoc and M. Nyk, J. Mater. Chem. C, 2015, 3, 5332-5338.

47 P. Haro-Gonzalez, W. T. Ramsay, L. M. Maestro, B. del Rosal, K. Santacruz-Gomez, M. D. Iglesias-de la Cruz, F. Sanz-Rodriguez, J. Y. Chooi, P. R. Sevilla, M. Bettinelli, D. Choudhury, A. K. Kar, J. G. Sole, D. Jaque and L. Paterson, Small, 2013, 9, 2162-2170.

48 J. T. Hu, L. S. Li, W. D. Yang, L. Manna, L. W. Wang and A. P. Alivisatos, Science, 2001, 292, 2060-2063.

49 J. Zhou, G. Chen, E. Wu, G. Bi, B. Wu, Y. Teng, S. Zhou and J. Qiu, Nano Lett., 2013, 13, 2241-2246.

50 G. Dong, H. He, Q. Pan, G. Chen, J. Xie, Z. Ma and M. Peng, ChemPlusChem, 2014, 79, 601-609.

51 P. Chen, M. Song, E. Wu, B. Wu, J. Zhou, H. Zeng, X. Liu and J. Qiu, Nanoscale, 2015, 7, 6462-6466.

52 K. Binnemans, Coord. Chem. Rev., 2015, 295, 1-45.

53 P. Haro-Gonzalez, B. del Rosal, L. M. Maestro, E. Martin Rodriguez, R. Naccache, J. A. Capobianco, K. Dholakia, J. G. Sole and D. Jaque, Nanoscale, 2013, 5, 1219212199.

54 H. Rodríguez-Rodríguez, P. Rodríguez Sevilla, E. Martín Rodríguez, D. H. Ortgies, M. Pedroni, A. Speghini, M. Bettinelli, D. Jaque and P. Haro-González, Small, 2015, 11, 1555-1561. 